\title{
Reconfigurable cluster-state generation in specially poled nonlinear waveguide arrays
}

\author{
James G. Titchener, ${ }^{1,2, *}$ Alexander S. Solntsev, ${ }^{1,3, \dagger}$ and Andrey A. Sukhorukov ${ }^{1, \ddagger}$ \\ ${ }^{1}$ Nonlinear Physics Centre, Research School of Physics, Australian National University, Canberra, ACT 2601, Australia \\ ${ }^{2}$ Quantum Technology Enterprise Centre, Quantum Engineering Technology Labs, H. H. Wills Physics Laboratory and Department of \\ Electrical and Electronic Engineering, University of Bristol, BS8 1FD, United Kingdom \\ ${ }^{3}$ School of Mathematical and Physical Sciences, University of Technology Sydney, 15 Broadway, Ultimo NSW 2007, Australia
}

(Received 2 March 2019; accepted 2 January 2020; published 7 February 2020)

\begin{abstract}
We present an approach for generating cluster states on-chip, with the state encoded in the spatial component of the photonic wave function. We show that for spatial encoding, a change of measurement basis can improve the practicality of cluster-state algorithm implementation and demonstrates this by simulating the Grover's search algorithm. Our state generation scheme involves shaping the wave function produced by spontaneous parametric down-conversion in on-chip waveguides using specially tailored nonlinear poling patterns. Furthermore, the form of the cluster state can be reconfigured quickly by driving different waveguides in the array.
\end{abstract}

DOI: 10.1103/PhysRevA.101.023809

\section{INTRODUCTION}

Cluster states are highly entangled, multiparticle quantum states [1] that have drawn significant interest for their potential in quantum information processing [2,3]. These multiqubit states form a complete basis for one-way quantum computation, where algorithms are carried out by successive measurement of qubits, causing information to flow through the state via entanglement $[4,5]$. Crucially for practical applications, cluster states have been shown to be robust to decoherence and loss of qubits [6]. In solid-state physics, cluster states are naturally produced in spin lattices interacting by an Isingtype Hamiltonian $[1,7,8]$, but increasingly they are considered useful in quantum photonic systems [9].

In quantum photonics the nonlinear interactions required for gates between single photons are challenging to realize [10], while measurement-based approaches to quantum computation, including those employing cluster states, can be more readily implemented [11]. Cluster states based on photonic polarization qubits have been generated in bulk optical systems utilizing nonlinear optics [12-18] or periodically driven quantum dots [19] to achieve the required entanglement between multiple photons. The basic elements of quantum computation have been demonstrated with these polarization qubit states, including qubit rotation, two-qubit gates, and small-scale versions of algorithms, such as Grover's algorithm [12]. Photonic cluster states can also be created using continuous-variable quantum entanglement [20-22], where the qubits are encoded in the time-dependent quadrature of the field. Furthermore, large cluster states have been demonstrated on-chip using frequency space encoding of the qubits [23]. Here we consider the generation of cluster states

\footnotetext{
*j.g.titchener@gmail.com

†alexander.solntsev@uts.edu.au

\$andrey.sukhorukov@anu.edu.au
}

using a fully spatial encoding of each qubit, which is well suited for on-chip implementation.

Typically photonic cluster states are generated in bulk optical setups by passing a pulsed pump laser twice through a nonlinear crystal, generating a pair of photons by spontaneous parametric down-conversion (SPDC) on each pass, giving a four-photon polarization entangled state [12]. Whereas up to 12-photon entanglement was reported recently [24,25], realization of multiphoton cluster states remains a challenging problem. Alternatively, just two photons can be used, since by exploiting hyperentanglement in spatial and polarization degrees of freedom multiple qubits can be encoded into each photon $[13,16]$. This approach has the advantage of reaching higher photon count rates while still producing nontrivial four-qubit cluster states. Encoding multiple qubits in a single photon has the potential to significantly increase the size of cluster states that can be realized, and it has been shown that such states can be used for quantum algorithms [13]. However, it is important to note that having multiple qubits encoded into a single photon decreases the flexibility of the overall system to execute arbitrary algorithms, since pairs of qubits encoded into a single photon must always be measured at the same time. Furthermore, for each extra qubit encoded into a photon via this method the number of modes the photon can occupy must be doubled, which is impractical beyond a small number of extra qubits per photon.

So far the realization of spatially encoded cluster states has been largely restricted to bulk optical setups, but inevitably, scalable cluster-state generation will require full onchip integration. Cluster states based on hyperentanglement between polarization and spatial degrees of freedom have been demonstrated on-chip [17], but a more natural and convenient realization would be based on just the spatial degree of freedom, since processing orthogonal polarizations in the same waveguide requires highly specialized fabrication platforms. In principle, existing silicon photonics approaches [26] could generate spatially encoded cluster states through use of reconfigurable linear optics to tune the wave function 
created by on-chip photon sources. However, it is interesting to consider the design of nonlinear sources which directly generate cluster states from a nonlinear interaction with no linear optical postprocessing step.

Here, we describe a method for the generation of cluster states within a nonlinear photonic chip with no reconfigurable elements where the state is fully encoded in the spatial properties of the photons. This method allows switching between different cluster states all optically, without a need for complex reconfigurable components to be integrated on-chip. The paper is organized as follows. In Sec. II we introduce our general method for the generation of spatially entangled photon states in arrays of coupled nonlinear waveguides. Then, in Sec. III we specify how the domain poling patterns in the waveguides can be designed to produce cluster states. In Sec. IV we demonstrate how simple computations could be carried out on the generated cluster states. Finally, we present conclusions and outlook in Sec. V.

\section{NONLINEAR SPATIAL ENTANGLEMENT GENERATION}

We consider a photonic chip with second-order nonlinearity so that pairs of photons can be generated via type-I spontaneous parametric down-conversion [27-30]. It has been shown that SPDC in arrays of coupled waveguides provides a stable source of highly entangled two-photon states [31] and that $\chi^{(2)}$ poling in the array can be engineered to produce tailored two-photon quantum states [32]; thus it is a natural platform to consider for the generation of cluster states. Similarly to Refs. $[13,16]$, we propose to use two-photon states to encode four-qubit cluster states. However, instead of exploiting hyperentanglement between polarization and spatial modes, we will use spatially distributed entanglement across an array.

We demonstrate a potential for cluster-state generation in an array of eight nonlinear waveguides, where specially tailored $\chi^{(2)}$ poling allows the production of specific twophoton states via SPDC. We consider the regime where the signal and idler photons are indistinguishable spectrally, while their state is engineered (via the nonlinear poling pattern) such that observing one photon in an odd-numbered waveguide guarantees the other is in an even-numbered waveguide (i.e., perfect spatial antibunching). Thus the system consists of two photons, each with four states available to it, giving a total of 16 distinct two-photon states. As shown in Fig. 1, each of these two-photon states can be mapped to a different four-qubit state by encoding two computational qubits into the state of each photon. For example, the physical twophoton state $|1\rangle_{\text {odd }}|2\rangle_{\text {even }}$, with one photon in waveguide 1 and the other in waveguide 2, would correspond to the fourqubit state $|0\rangle_{1}|0\rangle_{2}|0\rangle_{3}|0\rangle_{4}$. Here the state of computational qubits $|\ldots\rangle_{1}$ and $|\ldots\rangle_{2}$ is defined by the physical state of the down-converted photon in the four odd-numbered waveguides $\left(|\ldots\rangle_{\text {odd }}\right)$, and qubits $|\ldots\rangle_{3}$ and $|\ldots\rangle_{4}$ are defined by the state of the other down-converted photon in the four even-numbered waveguides. Thus four-qubit cluster states can be generated in the eight-waveguide system when the two-photon spatial wave function is shaped accordingly.

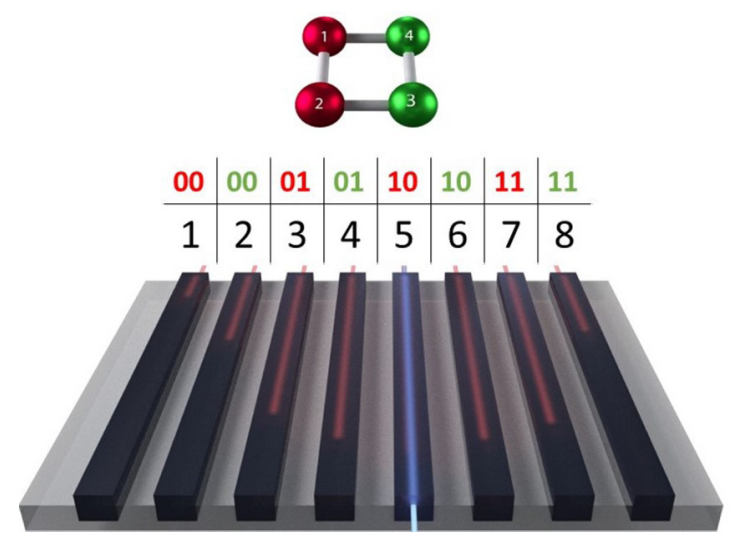

FIG. 1. Conceptual diagram of a cluster state encoded in an array of eight waveguides. The array is driven by a pump laser (blue) producing a pair of entangled photons (red). The encoding from the two-photon eight-waveguide state to a four-qubit cluster state is shown in the table, whereby a photon in the odd-numbered waveguides represents the first two binary qubits of the four-qubit cluster state (red-cluster-state qubits), and the photon in the evennumbered waveguides represents qubits 3 and 4 (green).

\section{DOMAIN POLING AND CLUSTER STATES}

In order to shape the wave function in the array, we propose to use tailored domain poling patterns to allow control of the local effective nonlinearity and thereby define the local phase of SPDC photon-pair generation at different points along the pumped waveguide. In combination with the continuous photon-pair coupling to neighboring waveguides, this allows tailoring of the output spatial wave function. Similar methods have allowed wave function engineering in specially poled bulk nonlinear crystals [33-35] and in arrays of up to four coupled waveguides [32,36]. Here we show how such control of the wave function can be achieved in eight coupled waveguides using only a single size of inverted $\chi^{(2)}$ domains, making fabrication of the structures feasible to implement with existing technology.

Using special domain poling patterns to control the twophoton wave function can be preferable to adjusting the linear properties of the chip such as the interwaveguide coupling rate. This is because poling structures affect only the nonlinear interaction, whereas the interwaveguide coupling rate affects both linear and nonlinear properties of the chip. With our approach each waveguide can be given a different nonlinear poling pattern to produce a different state; see the mathematical formulation in Appendix A. This allows the chip to be quickly reconfigured to produce different cluster states simply by driving different waveguides with the pump laser. This also avoids the need to integrate complex thermal or electrooptic phase shifters onto the chip to reconfigure the wave function. Thus, inhomogeneous waveguide poling provides a straightforward approach to generating and reconfiguring different photonic wave functions on-chip.

To this end we develop a class of nonlinear poling patterns that give precise control over the local effective nonlinear coefficient of each waveguide; see the mathematical details in Appendix B. Particularly, we focus on designing patterns that would be easy to fabricate, thus avoiding varied domain sizes 

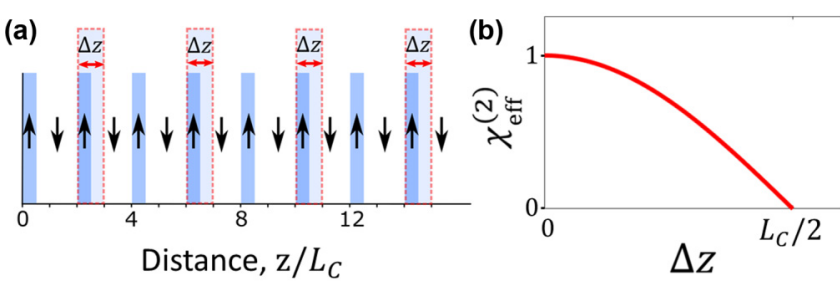

FIG. 2. (a) Diagram of the poling technique. Every second "up" domain can be translated by $\Delta z$ to alter the local effective nonlinearity. (b) The local effective nonlinearity (normalized to unity) vs the domain translation $(\Delta z)$, showing full control with a translation of half a coherence length $\left(L_{C} / 2\right)$.

such as in Refs. [32,34]. This is achieved by superimposing two fourth-order periodic poling patterns [Fig. 2(a)]. Fourthorder patterns have a period equal to 4 times the decoherence length of the SPDC process $\left(4 L_{C}\right)$, and in this case we consider patterns where the "up" domain length is $0.5 L_{C}$ and the remaining $3.5 L_{C}$ is poled down. Two of these patterns are then superimposed, as in Fig. 2(a), to make a second-order phase-matched poling pattern. The displacement between the two fourth-order patterns, $\Delta z$, determines the phase difference between the two-photon wave function generated from each poling pattern. Accordingly, the local effective nonlinearity of the poling structure can be controlled by varying this displacement as shown in Fig. 2(b). Translating the whole structure (with respect to other sections of poling on the waveguide) changes the overall phase of the wave function generated from that section of poling. Therefore the displacement of overlapping fourth-order patterns with respect to one another $(\Delta z)$ controls the magnitude of the effective nonlinearity, while translating the whole structure controls the phase.

A key consideration for experimental realization of these poling patterns is the tolerance of the output two-photon wave function to fabrication errors in the poling structure. Previous work simulated random errors in domain-boundary location and found that high-fidelity states could still be produced, despite Gaussian errors in all domain positions with standard deviation $1 \mu \mathrm{m}$ [32]. However, the output state would not be so robust to systematic errors in the domain-boundary locations. These are likely to occur because the growth of ferroelectric domains is a complex process, and fabrication parameters such as electrode size must be determined empirically to produce the required domain size [37]. Thus it is difficult to fabricate domains with a range of different sizes on the chip, and domains spaced too closely together can interact or fuse together during the inversion process, producing systematic errors. We avoid these systematic errors by using a poling pattern where all inverted domains are the same size, and no two domains are spaced so closely that they interact during the domain inversion process, as can be seen in Fig. 2(a).

In order to create tailored wave functions using this poling technique, we divide each waveguide in the nonlinear waveguide array into 34 different sections and allow each section to have a different poling pattern of the form shown in Fig. 2(a), and thus a different effective nonlinearity. Through algorithmic optimization of the effective nonlinearity in each
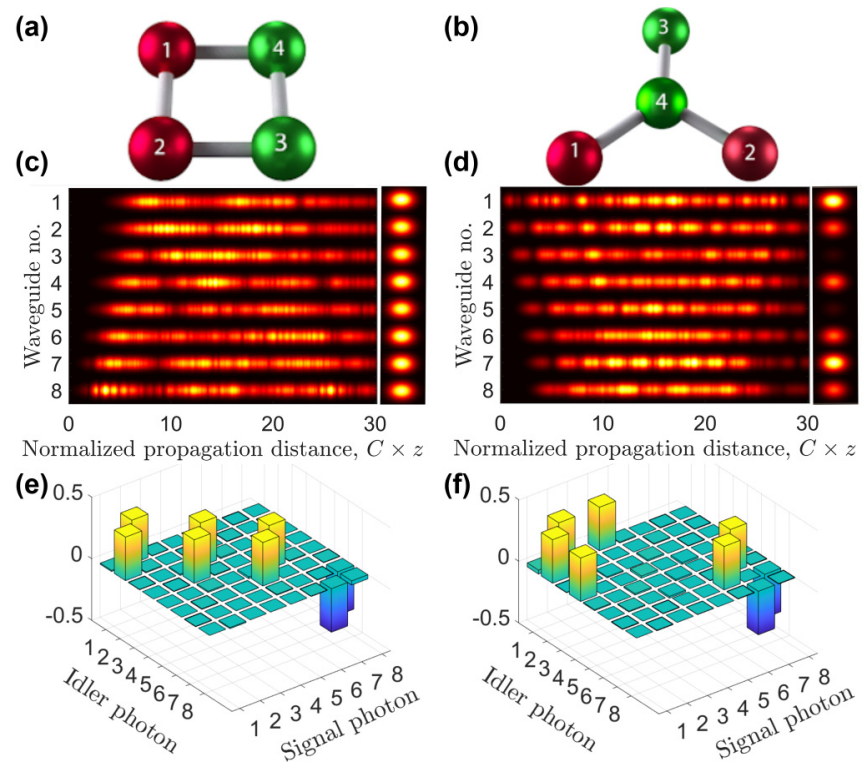

FIG. 3. (a), (b) Structure of the box and star cluster states, where red (green) spheres represent qubits physically encoded in the signal (idler) photon. (c), (d) Average number of down-converted photons in each waveguide during the generation of the box and star cluster states, respectively, using special waveguide array poling described in Fig. 2. (e), (f) Corresponding output two-photon wave functions produced when pumping waveguides 8 and 1 of the array, where (e) has a fidelity of $99.8 \%$ to the perfect box cluster state and (f) has a fidelity of $99.9 \%$ to a perfect star cluster state.

section, we can design tailored poling structures to produce, via SPDC, a desired two-photon state at the output of the array. For a practical source of cluster states we design an eight-waveguide device that produces the box or star cluster states [Figs. 3(a) and 3(b)] when waveguides number 8 or 1 , respectively, are driven by the pump laser. The simulated average number of down-converted photons along each waveguide in the device is presented in Figs. 3(c) and 3(d) for generation of the box and star cluster states, respectively. The full output wave functions produced from the poling structures are presented in Figs. 3(e) and 3(f), with fidelity to the ideal box and star cluster states of $99.8 \%$ and $99.9 \%$, respectively. Note that, relative to the usual representation, the cluster states shown here have a Hadamard transformation applied to each qubit for reasons that are explained in the following section.

\section{CLUSTER-STATE ALGORITHMS}

Once the photons are created, cluster-state computation algorithms proceed by sequentially measuring different qubits in the state. The basis used to measure qubit number $i$ is denoted $B_{i}(\alpha)$, with basis states $|\psi( \pm \alpha)\rangle_{i}=$ $\left(|0\rangle_{i} \pm e^{i \alpha}|1\rangle_{i}\right) / \sqrt{2}$, where the value of $\alpha$ is adjusted for each qubit measurement, depending on the algorithm being implemented [3]. In the context of a waveguide array this measurement basis is nontrivial to implement because it requires spatial transformations on the output waveguides to rotate to the $B(\alpha)$ measurement basis, regardless of the value of $\alpha$. However, we observe that for many simple operations, such as propagating a state through a circuit or performing a 
controlled-NOT CNOT gate, only measurements in the bases $B(0)$ and $B(\pi)$ are required [3,5]. Under a Hadamard transformation, these bases are mapped to direct measurements in the waveguide mode basis, i.e., $\hat{\boldsymbol{H}}\left(|0\rangle_{i}-|1\rangle_{i}\right) / \sqrt{2}=|1\rangle_{i}$ and $\hat{\boldsymbol{H}}\left(|0\rangle_{i}+|1\rangle_{i}\right) / \sqrt{2}=|0\rangle_{i}$, so there is no need to perform any linear transformation before measurement. Thus in order to implement spatially encoded cluster-state algorithms more efficiently in our proposed encoding scheme, a Hadamard transformation should be applied to all the measurement bases used for the algorithm. Additionally, to preserve the form of the algorithm, Hadamard transformations should be applied to each qubit in the cluster states itself. Thus the cluster states we designed above are the typical cluster states but with a Hadamard transformation applied to each qubit in the state.

After Hadamard transformations are applied to each qubit of the star cluster state, the resulting wave function is

$$
C_{4}^{\text {star }}=|+\rangle_{1}|+\rangle_{2}|+\rangle_{3}|0\rangle_{4}+|-\rangle_{1}|-\rangle_{2}|-\rangle_{3}|1\rangle_{4},
$$

where $| \pm\rangle_{i}=|0\rangle_{i} \pm|1\rangle_{i}$. The corresponding spatial twophoton wave function of this state is shown in Fig. 3(f). Such a state could be used to implement a CNOT gate [3], provided the measurement basis is the Hadamard transformation of the typical basis. Similarly, we define the box cluster state as

$$
\begin{aligned}
C_{4}^{\text {box }}= & |+\rangle_{1}|+\rangle_{2}|+\rangle_{3}|+\rangle_{4}+|+\rangle_{1}|-\rangle_{2}|+\rangle_{3}|-\rangle_{4} \\
& +|-\rangle_{1}|+\rangle_{2}|-\rangle_{3}|+\rangle_{4}-|-\rangle_{1}|-\rangle_{2}|-\rangle_{3}|-\rangle_{4},
\end{aligned}
$$

and the representation of this state as a two-photon spatial wave function is shown in Fig. 3(e).

The box cluster state can be used for an implementation of Grover's search algorithm [12-14,38]. For the simple case of a two-qubit database, this search consists of two steps. First, a two-bit state is prepared in the $|+\rangle|+\rangle$ state, and a two-bit string to be recovered (e.g., 01) is encoded into the state by inverting the sign of the corresponding wave-function element (e.g., $|0\rangle|1\rangle$ ). In the next step, the amplitude of the quantum state representing this encoded string is amplified by inverting the entire state about the mean. For the two-qubit case the answer is produced in a single iteration.

The measurements required to implement this algorithm using the box cluster state are shown in Fig. 4(a), with our implementation in the eight-waveguide spatial encoding shown in Fig. 4(b). As discussed above, for spatially encoded cluster states we propose to use a measurement basis that is the Hadamard transform of the usual measurement basis. We now define the basis explicitly as $B_{i}^{\hat{H}}(\alpha)$ with basis states $|\psi( \pm \alpha)\rangle_{i}=\left(|+\rangle_{i} \pm e^{i \alpha}|-\rangle_{i}\right) / \sqrt{2}$, where detection of one of the two basis states is interpreted as a logical 0 or 1 , respectively. To implement the Grover's search algorithm in this spatial encoding, at first the qubits 1 and 2 are measured, which physically involves detecting which odd-number waveguide the signal photon is in. The choice of measurement basis determines the bit string that is marked for recovery. Measuring qubit $i \in\{1,2\}$ in the basis $B_{i}^{\hat{H}}(\pi)$ [or $\left.B_{i}^{\hat{H}}(0)\right]$ will encode a logical 0 (or 1) into the $i$ th element of the bit string to be recovered. If both measurement results, $s_{1}$ and $s_{2}$, are 0 , the initial encoding of the two-bit string was successful; otherwise unsuccessful encoding can be compensated for by feeding forward the measurement results and using them to rotate the measurement bases for qubits 3 and 4 . To recover the encoded

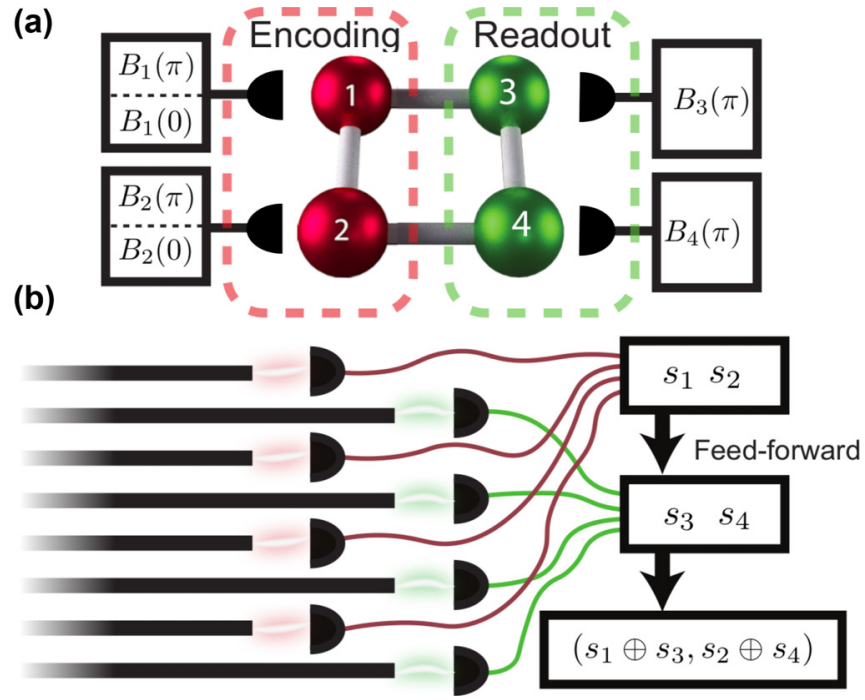

FIG. 4. Operation of Grover's search algorithm: (a) cluster-state diagram of the implementation of Grover's search and (b) implementation in the eight-waveguide system. Measurements are made directly in the waveguide output mode basis.

bit string via Grover's search algorithm, the remaining two qubits, 3 and 4, are measured in the basis $B_{i}^{H}(\pi)$, physically achieved by detecting the idler photon in one of the evennumbered waveguides. In place of rotating the measurement basis of qubits 3 and 4, postprocessing of results can instead be used, reinterpreting the final result as $\left(s_{1} \oplus s_{3}, s_{2} \oplus s_{4}\right)$. This recovers the marked bit string with certainty.

\section{CONCLUSION AND OUTLOOK}

In conclusion, we have shown how to design a nonlinear photonic chip to generate and optically switch between different four-qubit cluster states. This is achieved using a nonlinear waveguide array with specially tailored poling patterns, which are optimized to be easy to fabricate with typical electric field poling methods. Importantly, this can provide a stable integrated source of cluster states, with potential to scale to larger states by increasing the number of waveguides in the array. We also propose a change of measurement basis to implement the cluster-state algorithms with spatially encoded photonic qubits.

Clearly, nontrivial demonstrations of cluster-state quantum computing will require resource states of significantly more than four entangled qubits. To achieve this, simple clusterstate sources such as the one proposed here must be multiplexed in time, space, or both. However, spatial multiplexing via cluster-state fusion operations [9] requires destructive measurement of a photon in each input state; thus at least three photons are required in each input state for fusion to produce a cluster state with more qubits than either of the input states. Therefore, to make a scalable cluster-state source, it is worth considering extending the technique presented here to the generation of four-photon states via $\chi^{(2)}$ nonlinearity so that fusion operations could be applied. Another promising route to scalability is via temporal multiplexing of cluster states generated sequentially by a pulsed pump. The temporal 
multiplexing has been demonstrated for continuous-variable (CV) states $[39,40]$. The generation of CV states within photonic chips has recently been realized [41], and we anticipate that nonlinear poling techniques in waveguide arrays might be also applied to shape the spatial entanglement of CV states which could subsequently be multiplexed in time to create large multidimensional cluster states.

\section{ACKNOWLEDGMENTS}

We acknowledge funding from the Australian Research Council (ARC) through Projects No. DP160100619, No. DP190100277, and No. DE180100070. The authors thank Mirko Lobino for valuable discussions.

\section{APPENDIX A: SPDC IN A POLED WAVEGUIDE ARRAY}

In the main text we have described how custom nonlinear poling patterns could be used to generate two-photon cluster states. This Appendix provides complete mathematical details on the poling patterns used in the main text. We follow the general framework of Ref. [32] but introduce special aspects in the analysis to ensure that the poling patterns are practical to fabricate using typical electric field poling with lithographically defined electrode masks.

The two-photon wave function at position $z$ along the propagation axis of the $\chi^{(2)}$ waveguide array obeys the equation $[31,32]$

$$
\begin{aligned}
i \frac{\partial \Psi_{n_{s}, n_{i}}(z)}{\partial z}= & i \sum_{n_{p}=1}^{N} A_{n_{p}} d_{n_{p}}(z) e^{i \Delta \beta^{(0)} z} \delta_{n_{s}, n_{p}} \delta_{n_{i}, n_{p}} \\
& -C\left[\Psi_{n_{s}, n_{i}+1}+\Psi_{n_{s}, n_{i}-1}+\Psi_{n_{s}+1, n_{i}}+\Psi_{n_{s}-1, n_{i}}\right] .
\end{aligned}
$$

Here $n_{s}$ and $n_{i}$ are the waveguide numbers of the signal and idler photons, $N$ is the total number of waveguides, the first term on the right-hand side represents the generation of the photon pair via SPDC, with classical laser driving amplitude $A_{n_{p}}$ and second-order nonlinear coefficient $d_{n_{p}}(z)$ in waveguide number $n_{p}$, and the phase mismatch of the nonlinear process is $\Delta \beta^{(0)}$. The last term on the right describes the evanescent coupling of signal and idler photons between neighboring waveguides with the coupling rate given by $C$. Integrating this equation along the length $(L)$ of the array in the basis of eigenmodes of the nearest coupling operator gives

$$
\begin{aligned}
f_{k_{s}, k_{i}}(L)= & e^{i \beta_{k_{s} k_{i}} L} \sum_{n_{p}=1}^{N} A_{n_{p}} \sin \left(\frac{\pi k_{i} n_{p}}{N+1}\right) \\
& \times \sin \left(\frac{\pi k_{s} n_{p}}{N+1}\right) \int_{0}^{L} d_{n_{p}}(z) e^{i\left(\Delta \beta^{(0)}-\beta_{k_{s} k_{i}}\right) z} d z .
\end{aligned}
$$

Here $k_{s}$ and $k_{i}$ are the indices for the two-photon eigenmodes, $\beta_{k_{s} k_{i}}=2 C\left[\cos \left(\frac{\pi k_{i}}{N+1}\right)+\cos \left(\frac{\pi k_{s}}{N+1}\right)\right]$, the contribution to the phase mismatch term resulting from photon coupling between waveguides. Following the approach in [32], we note that typically $\beta^{(0)} \gg \beta_{k_{s} k_{i}}$, meaning that to a good approximation the integral from Eq. (A2) can be separated into quickly and slowly varying terms according to

$$
\sum_{m=1}^{L / \Lambda} e^{-i\left(\beta_{k s k_{i}} m \Lambda\right)} \frac{1}{\Lambda} \int_{(m-1) \Lambda}^{m \Lambda} d_{n_{p}}(z) e^{i \Delta \beta^{(0)} z} d z .
$$

Here $\Lambda$ is a length equal to a small integer number multiple of the decoherence length $L_{C}$ (where by definition $L_{C}=$ $\left.2 \pi / \Delta \beta^{(0)}\right)$. We will choose $\Lambda=4 L_{C}$ for our structure. Note that $L / \Lambda$ is the total length of the array divided by $\Lambda$. For simplicity, we will assume the total length of the array is such that this quotient will produce an integer, i.e., the length of the array will fit a whole number of lengths $\Lambda$.

The slowly varying integral approximation according to Eq. (A3) allows Eq. (A2) to be written as

$$
\begin{aligned}
f_{k_{s}, k_{i}}(L)= & e^{i \beta_{k_{s} k_{i} L}} \sum_{n_{p}=1}^{N} A_{n_{p}} \sin \left(\frac{\pi k_{i} n_{p}}{N+1}\right) \\
& \times \sin \left(\frac{\pi k_{s} n_{p}}{N+1}\right) \sum_{m=1}^{L / \Lambda} D_{n_{p}}^{m} e^{-i\left(\beta_{k s k_{i}}\right) m \Lambda},
\end{aligned}
$$

where $D_{n_{p}}^{m}$ is the integral of the quickly varying term from Eq. (A3), given by

$$
D_{n_{p}}^{m}=\frac{1}{\Lambda} \int_{(m-1) \Lambda}^{m \Lambda} d_{n_{p}}(z) e^{i \Delta \beta^{(0)} z} d z .
$$

We refer to $D_{n_{p}}^{m}$ as the aggregate nonlinearity, since it gives the net effect of poling and phase mismatch for a section of waveguide from distance $(m-1) \Lambda$ to distance $m \Lambda$ along waveguide number $n_{p}$.

As we demonstrate below in Appendix B, for poling patterns of "up" and "down" ferroelectric domains [i.e., where $d_{n_{p}}(z)$ is restricted to values of $\left.\pm \chi^{(2)}\right]$, the aggregate nonlinearity $\left|D_{n_{p}}^{m}\right|$ can take any value less than the maximum of $(2 / \pi) \chi^{(2)}$, and any phase can be achieved simply by translating the poling pattern in the $z$ direction (with a cyclic shift at the boundaries).

Therefore in Eq. (A4) we can treat $D_{n_{p}}^{m}$ as a matrix of any set of complex numbers and optimize this matrix to produce the desired wave function $f_{k_{s}, k_{i}}(L)$ in the waveguide array eigenmode basis. This optimization can be carried out computationally by vectorizing Eq. (A4). Vectorization is best done by assuming only a single waveguide is pumped by the laser, so that $A_{n_{p}}=0$ for all $n_{p}$ except the pumped waveguide, $n_{p}^{\prime}$. Then Eq. (A4) can be written as

$$
\begin{aligned}
& f_{k_{s}, k_{i}}(L)=e^{i \beta_{k_{s} k_{i}} L} A_{n_{p}^{\prime}} \sin \left(\frac{\pi k_{i} n_{p}^{\prime}}{N+1}\right) \sin \left(\frac{\pi k_{s} n_{p}^{\prime}}{N+1}\right) \\
& \times \sum_{m=1}^{L / \Lambda} D_{n_{p}^{\prime}}^{m} e^{-i\left(\beta_{k_{s} k_{i}}\right) m \Lambda} \text {. }
\end{aligned}
$$

Here $D_{n_{p}^{\prime}}^{m}$ is interpreted as a $(L / \Lambda) \times 1$ vector while the remaining terms are a $N^{2} \times(L / \Lambda)$ matrix, i.e.,

$$
f_{k}=\sum_{m=1}^{L / \Lambda} M_{k, m} D_{m}
$$


where $M_{k, m}$ represents all variables on the left of Eq. (A6) except $D_{n_{p}^{\prime}}^{m}$, and $k$ is an index concatenating all $N^{2}$ permutations of the eigenmode indices $k_{s}, k_{i}$ into a single index. This matrix can be inverted numerically to give the vector of aggregate nonlinearities $\left(D_{m}\right)$ required to produce a certain output wave function.

Note that so far, as defined in Eq. (A4), we have allowed the aggregate nonlinearity to change along the propagation axis in discrete steps every distance of $\Lambda$. However, this is typically an unnecessarily large number of free parameters compared to the number of parameters required to solve Eq. (A7) for arbitrary wave function $\Psi_{n_{s}, n_{i}}(L)$, since the number of elements in the two-photon wave function are of the order of $N^{2}$. Thus, we restrict $D_{m}$ to be constant within particular regions along the waveguide to simplify the final poling pattern. In the case considered in this paper, where we have eight waveguides, the laser-driven waveguide is divided into 34 different regions of constant aggregate nonlinearity. This provides enough free parameters in Eq. (A6) to produce the desired two-photon cluster states.

\section{APPENDIX B: DESIGN OF NONLINEAR POLING PATTERNS}

Once the required aggregate nonlinearity is known, Eq. (A5) can be used to find a suitable poling structure. One way to do this is to simply vary the duty cycle of the poling pattern [32]. However, as explained in the main text, here we aim to find poling patterns that would be easy to create using standard electric-field-induced poling using lithographically defined electrode masks. Two key considerations for the practicality of complex poling patterns are as follows. First, the inverted domains should have a constant size throughout the chip because the relationship between electrode size and poled domain size is complex. Secondly, two inverted domains should not be too close together (say closer than $L_{C} / 2$ ), since the domains could interact during the electric field poling process, producing errors in the final poling pattern.

With these constraints in mind we used a poling pattern consisting of two fourth-order patterns superimposed as illustrated in Fig. 2(a). The location of the two "up" domains in each period of the poling structure shown in Fig. 2(a) is mathematically defined as follows:

$$
\begin{aligned}
& \text { if } m L_{C}+z_{1}<z<(m+0.5) L_{C}+z_{1}, \\
& \text { then } d_{n_{p}}(z)=+\chi^{(2)}, \\
& \text { if }(m+2) L_{C}+z_{2}<z<(m+2.5) L_{C}+z_{2}, \\
& \text { then } d_{n_{p}}(z)=+\chi^{(2)},
\end{aligned}
$$

$$
\text { otherwise } d_{n_{p}}(z)=-\chi^{(2)} \text {. }
$$

Here $m$ is allowed to be only multiples of 4 (i.e., $0,4,8 \ldots$ ), since the poling pattern has a period of four decoherence lengths. The terms $z_{1}$ and $z_{2}$ are translations of each of the two inverted domains along the propagation axis. We restrict $-L_{C} \leqslant z_{2}-z_{1} \leqslant L_{C}$ to prevent the inverted domains from getting too close together.

The aggregate nonlinearity produced by the poling structure specified above can be found by integrating $d_{n_{p}}(z)$ over a length of $4 L_{C}$ according to Eq. (A5). Dividing the integral into the poled up and down parts gives

$$
\begin{aligned}
D_{n_{p}}^{m}= & \frac{-\chi^{(2)}}{\Lambda} \int_{m L_{C}}^{(m+4) L_{C}} e^{i \Delta \beta^{(0)} z} d z \\
& +\frac{2 \chi^{(2)}}{\Lambda} \int_{m L_{C}+z_{1}}^{(m+0.5) L_{C}+z_{1}} e^{i \Delta \beta^{(0)} z} d z \\
& +\frac{2 \chi^{(2)}}{\Lambda} \int_{(m+2) L_{C}+z_{2}}^{(m+2.5) L_{C}+z_{2}} e^{i \Delta \beta^{(0)} z} d z,
\end{aligned}
$$

where the integration of the negative part of the poling has been carried out over the whole length ( 0 to $\left.4 L_{C}\right)$, and twice the positive parts added to simplify the calculation. Noting that $L_{C}=2 \pi / \Delta \beta^{(0)}$ and $\Lambda=4 L_{C}$, we obtain the following after integration:

$$
D_{n_{p}}^{m}=\frac{i \chi^{(2)}}{2 \pi}\left(e^{i 2 \pi z_{1} / L_{C}}+e^{i 2 \pi z_{2} / L_{C}}\right) .
$$

We now define the relative shift of up domains as $\Delta z=z_{2}-$ $z_{1}$ and obtain

$$
D_{n_{p}}^{m}=\frac{i \chi^{(2)}}{\pi} e^{i \pi\left(z_{1}+z_{2}\right) / L_{C}} \cos \left(\pi \Delta z / L_{C}\right) .
$$

We see that by adjusting $\Delta z$ in the range $\left[0, L_{C} / 2\right]$, any absolute value of $D_{n_{p}}^{m}$ can be achieved, below the maximum of $\chi^{(2)} / \pi$. Furthermore, by increasing both $z_{1}$ and $z_{2}$ by the same amount (in the range $\left[0, L_{C}\right]$ ) any phase shift of $D_{n_{p}}^{m}$ can be achieved, independently of the absolute value.

Thus the poling structure described above allows arbitrary variation of the aggregate nonlinearity while guaranteeing that the structure is practical to fabricate. Accordingly, to determine the poling pattern to produce a specific output wave function, we have solved Eq. (A7) to calculate the required aggregate nonlinearity and subsequently used Eq. (B6) to find the parameters of specific up or down poling structure required to achieve the aggregate nonlinearities.
[1] H. J. Briegel and R. Raussendorf, Persistent Entanglement in Arrays of Interacting Particles, Phys. Rev. Lett. 86, 910 (2001).

[2] R. Raussendorf and H. J. Briegel, A One-Way Quantum Computer, Phys. Rev. Lett. 86, 5188 (2001).

[3] R. Raussendorf, D. E. Browne, and H. J. Briegel, Measurementbased quantum computation on cluster states, Phys. Rev. A 68 , 022312 (2003).
[4] S. Takeda and A. Furusawa, Toward large-scale fault-tolerant universal photonic quantum computing, APL Photonics 4, 060902 (2019).

[5] M. A. Nielsen, Cluster-state quantum computation, Rep. Math. Phys. 57, 147 (2006).

[6] M. Hein, W. Dur, and H. J. Briegel, Entanglement properties of multipartite entangled states under the influence of decoherence, Phys. Rev. A 71, 032350 (2005). 
[7] O. Mandel, M. Greiner, A. Widera, T. Rom, T. W. Hansch, and I. Bloch, Controlled collisions for multi-particle entanglement of optically trapped atoms, Nature (London) 425, 937 (2003).

[8] B. P. Lanyon, P. Jurcevic, M. Zwerger, C. Hempel, E. A. Martinez, W. Dur, H. J. Briegel, R. Blatt, and C. F. Roos, Measurement-Based Quantum Computation with Trapped Ions, Phys. Rev. Lett. 111, 210501 (2013).

[9] M. Gimeno-Segovia, P. Shadbolt, D. E. Browne, and T. Rudolph, From Three-Photon Greenberger-Horne-Zeilinger States to Ballistic Universal Quantum Computation, Phys. Rev. Lett. 115, 020502 (2015).

[10] A. Feizpour, M. Hallaji, G. Dmochowski, and A. M. Steinberg, Observation of the nonlinear phase shift due to single postselected photons, Nat. Phys. 11, 905 (2015).

[11] M. A. Nielsen, Optical Quantum Computation Using Cluster States, Phys. Rev. Lett. 93, 040503 (2004).

[12] P. Walther, K. J. Resch, T. Rudolph, E. Schenck, H. Weinfurter, V. Vedral, M. Aspelmeyer, and A. Zeilinger, Experimental oneway quantum computing, Nature (London) 434, 169 (2005).

[13] K. Chen, C. M. Li, Q. Zhang, Y. A. Chen, A. Goebel, S. A. Chen, A. Mair, and J. W. Pan, Experimental Realization of OneWay Quantum Computing with Two-Photon Four-Qubit Cluster States, Phys. Rev. Lett. 99, 120503 (2007).

[14] R. Prevedel, P. Walther, F. Tiefenbacher, P. Bohl, R. Kaltenbaek, T. Jennewein, and A. Zeilinger, High-speed linear optics quantum computing using active feed-forward, Nature (London) 445, 65 (2007).

[15] Y. Tokunaga, S. Kuwashiro, T. Yamamoto, M. Koashi, and N. Imoto, Generation of High-Fidelity Four-Photon Cluster State and Quantum-Domain Demonstration of One-Way Quantum Computing, Phys. Rev. Lett. 100, 210501 (2008).

[16] H. Wunderlich, G. Vallone, P. Mataloni, and M. B. Plenio, Optimal verification of entanglement in a photonic cluster state experiment, New J. Phys. 13, 033033 (2011).

[17] M. A. Ciampini, A. Orieux, S. Paesani, F. Sciarrino, G. Corrielli, A. Crespi, R. Ramponi, R. Osellame, and P. Mataloni, Path-polarization hyperentangled and cluster states of photons on a chip, Light Sci. Appl. 5, e16064 (2016).

[18] I. Dhand, M. Engelkemeier, L. Sansoni, S. Barkhofen, C. Silberhorn, and M. B. Plenio, Proposal for Quantum Simulation Via All-Optically-Generated Tensor Network States, Phys. Rev. Lett. 120, 130501 (2018).

[19] I. Schwartz, D. Cogan, E. R. Schmidgall, Y. Don, L. Gantz, O. Kenneth, N. H. Lindner, and D. Gershoni, Deterministic generation of a cluster state of entangled photons, Science 354, 434 (2016).

[20] S. Yokoyama, R. Ukai, S. C. Armstrong, C. Sornphiphatphong, T. Kaji, S. Suzuki, J. Yoshikawa, H. Yonezawa, N. C. Menicucci, and A. Furusawa, Ultra-large-scale continuousvariable cluster states multiplexed in the time domain, Nat. Photon. 7, 982 (2013).

[21] M. Chen, N. C. Menicucci, and O. Pfister, Experimental Realization of Multipartite Entanglement of 60 Modes of a Quantum Optical Frequency Comb, Phys. Rev. Lett. 112, 120505 (2014).

[22] R. N. Alexander, P. Wang, N. Sridhar, M. Chen, O. Pfister, and N. C. Menicucci, One-way quantum computing with arbitrarily large time-frequency continuous-variable cluster states from a single optical parametric oscillator, Phys. Rev. A 94, 032327 (2016).
[23] M. Kues, C. Reimer, P. Roztocki, L. R. Cortes, S. Sciara, B. Wetzel, Y. B. Zhang, A. Cino, S. T. Chu, B. E. Little, D. J. Moss, L. Caspani, J. Azana, and R. Morandotti, On-chip generation of high-dimensional entangled quantum states and their coherent control, Nature (London) 546, 622 (2017).

[24] H. S. Zhong, Y. Li, W. Li, L. C. Peng, Z. E. Su, Y. Hu, Y. M. He, X. Ding, W. J. Zhang, H. Li, L. Zhang, Z. Wang, L. X. You, X. L. Wang, X. Jiang, L. Li, Y. A. Chen, N. L. Liu, C. Y. Lu, and J. W. Pan, 12-Photon Entanglement and Scalable Scattershot Boson Sampling with Optimal Entangled-Photon Pairs from Parametric Down-Conversion, Phys. Rev. Lett. 121, 250505 (2018).

[25] X. L. Wang, Y. H. Luo, H. L. Huang, M. C. Chen, Z. E. Su, C. Liu, C. Chen, W. Li, Y. Q. Fang, X. Jiang, J. Zhang, L. Li, N. L. Liu, C. Y. Lu, and J. W. Pan, 18-Qubit Entanglement with Six Photons' Three Degrees of Freedom, Phys. Rev. Lett. 120, 260502 (2018).

[26] X. G. Qiang, X. Q. Zhou, J. W. Wang, C. M. Wilkes, T. Loke, S. O'Gara, L. Kling, G. D. Marshall, R. Santagati, T. C. Ralph, J. B. B. Wang, J. L. O'Brien, M. G. Thompson, and J. C. F. Matthews, Large-scale silicon quantum photonics implementing arbitrary two-qubit processing, Nat. Photon. 12, 534 (2018).

[27] A. S. Solntsev, A. A. Sukhorukov, D. N. Neshev, and Y. S. Kivshar, Spontaneous Parametric Down-Conversion and Quantum Walks in Arrays of Quadratic Nonlinear Waveguides, Phys. Rev. Lett. 108, 023601 (2012).

[28] A. S. Solntsev and A. A. Sukhorukov, Path-entangled photon sources on nonlinear chips, Rev. Phys. 2, 19 (2017).

[29] R. Kruse, F. Katzschmann, A. Christ, A. Schreiber, S. Wilhelm, K. Laiho, A. Gabris, C. S. Hamilton, I. Jex, and C. Silberhorn, Spatio-spectral characteristics of parametric downconversion in waveguide arrays, New J. Phys. 15, 083046 (2013).

[30] Y. F. Bai, P. Xu, L. L. Lu, M. L. Zhong, and S. N. Zhu, Two-photon Anderson localization in a disordered quadratic waveguide array, J. Opt. 18, 055201 (2016).

[31] A. S. Solntsev, F. Setzpfandt, A. S. Clark, C. W. Wu, M. J. Collins, C. L. Xiong, A. Schreiber, F. Katzschmann, F. Eilenberger, R. Schiek, W. Sohler, A. Mitchell, C. Silberhorn, B. J. Eggleton, T. Pertsch, A. A. Sukhorukov, D. N. Neshev, and Y. S. Kivshar, Generation of Nonclassical Biphoton States through Cascaded Quantum Walks on a Nonlinear Chip, Phys. Rev. X 4, 031007 (2014).

[32] J. G. Titchener, A. S. Solntsev, and A. A. Sukhorukov, Generation of photons with all-optically-reconfigurable entanglement in integrated nonlinear waveguides, Phys. Rev. A 92, 033819 (2015)

[33] X. Q. Yu, P. Xu, Z. D. Xie, J. F. Wang, H. Y. Leng, J. S. Zhao, S. N. Zhu, and N. B. Ming, Transforming Spatial Entanglement using a Domain-Engineering Technique, Phys. Rev. Lett. 101, 233601 (2008).

[34] Y. Q. Qin, C. Zhang, Y. Y. Zhu, X. P. Hu, and G. Zhao, WaveFront Engineering by Huygens-Fresnel Principle for Nonlinear Optical Interactions in Domain Engineered Structures, Phys. Rev. Lett. 100, 063902 (2008).

[35] H. Y. Leng, X. Q. Yu, Y. X. Gong, P. Xu, Z. D. Xie, H. Jin, C. Zhang, and S. N. Zhu, On-chip steering of entangled photons in nonlinear photonic crystals, Nat. Commun. 2, 429 (2011). 
[36] F. Lenzini, A. N. Poddubny, J. Titchener, P. Fisher, A. Boes, S. Kasture, B. Haylock, M. Villa, A. Mitchell, A. S. Solntsev, A. A. Sukhorukov, and M. Lobino, Direct characterization of a nonlinear photonic circuit's wave function with laser light, Light Sci. Appl. 7, 17143 (2018).

[37] M. Houe and P. D. Townsend, An introduction to methods of periodic poling for 2nd-harmonic generation, J. Phys. D 28, 1747 (1995).

[38] L. K. Grover, Quantum Mechanics Helps in Searching for a Needle in a Haystack, Phys. Rev. Lett. 79, 325 (1997).

[39] J. Yoshikawa, S. Yokoyama, T. Kaji, C. Sornphiphatphong, Y. Shiozawa, K. Makino, and A. Furusawa, Invited article: Generation of one-million-mode continuous-variable cluster state by unlimited time-domain multiplexing, APL Photonics 1, 060801 (2016).

[40] W. Asavanant, Y. Shiozawa, S. Yokoyama, B. Charoensombutamon, H. Emura, R. N. Alexander, S. Takeda, J. Yoshikawa, N. C. Menicucci, H. Yonezawa, and A. Furusawa, Time-domain multiplexed 2-dimensional cluster state: Universal quantum computing platform, Science 366, 373 (2019).

[41] F. Lenzini, J. Janousek, O. Thearle, M. Villa, B. Haylock, S. Kasture, L. Cui, H. P. Phan, D. V. Dao, H. Yonezawa, P. K. Lam, E. H. Huntington, and M. Lobino, Integrated photonic platform for quantum information with continuous variables, Sci. Adv. 4, eaat9331 (2018). 